\title{
The Outcomes of Patients Incidentally Confirmed with Covid-19 After Cardiac Surgery
}

\author{
Ayhan Uysal, MD, ${ }^{1}$ Esra Ertürk, MD,${ }^{2}$ Ahmet Feyzi Abacilar, MD,${ }^{3}$ Umit Duman, MD, ${ }^{4}$ \\ Omer Faruk Dogan, MD $^{5}$
}

${ }^{1}$ Frrat University Medical Faculty Cardiovascular Surgery Clinic, Elazıg, Turkey; ${ }^{2}$ The Health Science University, Mersin City Hospital, Department of Cardiovascular Surgery, Mersin, Turkey; ${ }^{3}$ The Private Izmir Su Hospital, Department of Cardiovascular Surgery, Izmir, Turkey; ${ }^{4}$ Private Tekirdag Hospital, Department of Cardiovascular Surgery, Tekirdag, Turkey; ${ }^{5}$ Adiyaman University School of Medicine, Department of Cardiovascular Surgery, Adiyaman/Turkey

\section{ABSTRACT}

Background: The aim of this study was to investigate the clinical outcomes of cardiac surgery in patients who were incidentally diagnosed with Covid-19 in the postoperative period.

Patients and methods: We performed 826 open cardiac surgeries in five tertiary centers. Most of the surgeries were elective coronary artery bypass grafting (CABG) (93.8\%). A preoperative RT-PCR test and transcutaneous oxygen saturation were routinely investigated prior to surgery. We also investigated whether the patients already received Covid-19 treatment or had any contact with a Covid-19 patient in the last two weeks. We analyzed high sensitive C-reactive protein (hs-CRP), d-dimer, and fibrinogen, which plays a main role in the activation of procoagulant state after surgeries.

Results: Acute lung injury related to Covid-19 activation was observed in 48 out of 826 patients $(5.8 \%)$. The median age of 48 patients was $63.9 \pm 12.4$ years. Euro-Score and body mass index (BMI) were $6.1 \pm 1.1$ and $29.2 \pm 4.1 \mathrm{~kg} / \mathrm{m}^{2}$, respectively. RT-PCR test results were positive in 29 patients $(60.4 \%)$. We performed thoracic computed tomography (CT) in all patients with or without positive RT-PCR test results. Thoracic CT images showed that there was a different degree of ARDS (mild, moderate, and serious). The median time of extracorporeal circulation (ECC) was $93.2 \pm 14.6 \mathrm{~min}$. in on-pump surgery (IQR, 68-155 min.). Common symptoms included dyspnea $(N=22 ; 45.8 \%)$ and fever $(N=12 ; 25 \%)$. Eleven patients needed readmission to ICU. Compared with non-admitted to ICU patients, ICU patients were higher comorbidities and severe laboratory abnormalities (eg, high blood d-dimer and fibrinogen). We also detected significantly low oxygen saturation, hypercapnia, and severe acidosis in readmitted patients.

Received August 23, 2021; received in revised form September 7, 2021; accepted September 7, 2021.

Correspondence: Omer Faruk Dogan, MD, Department of Cardiovascular Surgery Adiyaman University School of Medicine, Training and Research Hospital Altinsehir No:1, Adiyaman, Turkey, Telephone +90(533)5334813056, Fax+90(416)2251010 (e-mail: ofdogan@bacettepe.edu.tr).
Radiologic investigations showed that there were severe ARDS with bilateral pneumonic infiltration resistant to medical treatment in 6 out of 11 patients who died (54.5\%).

Conclusion: Diffuse pneumonic infiltration related to Covid-19 may develop in asymptomatic cardiac surgery patients with negative RT-PCR test results. Immunologic disorders resulting from ECC, physiologic distress, and anesthesia may activate Covid-19 during incubation period. We need randomized clinical trials to explain Covid-19 activation in latent period of the virus, and clinical outcomes in cardiac surgery.

\section{INTRODUCTION}

The first case confirmed with Coronavirus-2019 (Covid19; SARS-CoV-2) was reported in December 2019 [Chen 2020]. Since the new coronavirus was spreading rapidly in many countries and has a high mortality rate, it was declared a pandemic by the World Health Organization [WHO 2019]. The Covid-19 pandemic presents many challenges for clinicians and surgeons with many unknown aspects and a high admission rate to ICU because of an ARDS [Welt 2020].

To prevent the spread of Covid-19, a number of scientific associations suggest that cardiac and non-cardiac surgeries should be classified as urgent, emergent, and elective [Ing 2020; Curzen 2020; Chieffo 2020]. Unfortunately, classifying cardiac surgery patients is a double-edged sword since they have some comorbid diseases, such as diabetes and hypertension, that increase the risk of Covid-19. Mortality and morbidity rates in patients confirmed with Covid-19 who had ST-segment elevation [Bangalore 2020; Giulio 2020] or cardiac surgery [Barkhordari 2020] have been reported in a limited number of reports. Barkhordari et al. reported that outcomes of urgent cardiac surgery in patients confirmed with Covid-19 were poor, and serious respiratory complications rates were high when compared with similar patients in the pre-pandemic period ( $2 \%$ vs. $16 \%$ ) [Barkhordari 2020]. Lei et al. demonstrated in their cohort of 34 non-cardiac surgery patients with confirmed Covid-19 that the mortality rate was 20\% [Lei]. Therefore, these authors proposed postponing elective non-cardiac and cardiac surgery. Aminian et al. presented non-cardiac surgeries in 4 patients who underwent cholecystectomy, hernia repair, gastric bypass, and hysterectomy in the perioperative course 
with diagnostic challenge and a high mortality in the initial period of Covid-19 [Aminian 2020].

Lymphopenia and high blood levels of CRP, high levels of interleukins, cardiac enzyme release, fibrinogen, and D-dimer were correlated with the postoperative hypoxemia as independent hospital mortality factors [Niknam 2020; Shi 2020; Keskin 2021; Keskin 2021]. Since we still are living in the pandemic period, there aren't sufficient studies for explaining the relationship between the latent period of Covid-19 and its activation postoperatively.

We present pulmonary and systemic complications as a result of postoperative Covid-19 activation in patients who had preoperative negative RT-PCR test results, as well as the mortality and morbidity after cardiac surgery.

\section{MATERIAL AND METHODS}

This retrospective study was performed in five tertiary centers (Adiyaman University School of Medicine, Mersin City Hospital, Tekirdağ Private Yaşam Hospital, The Private Izmir Su Hospital, and Firat University School of Medicine) from April 30, 2020, to July 1, 2021.

We confirmed Covid-19 RT-PCR test results 48 hours after cardiac surgery. Patients had no respiratory symptoms. Median Euro-Score and body mass index (BMI) in 48 patients were $6.1 \pm 1.1$ and $29.2 \pm 4.1 \mathrm{~kg} / \mathrm{m}^{2}$, respectively. Systemic hypertension and diabetes mellitus were the most common comorbidities. We also investigated whether the patients previously received Covid-19 treatment. We investigated the history of Covid-19 two weeks prior to surgery.

Inclusion criteria were having no preoperative symptoms related to Covid-19 (e.g. fever, dyspnea), preoperative negative RT-PCR test, and no history of contact with Covid-19 patients. We excluded hemodialysis patients, as well as those with active infection, history of malignancy, chronic liver disease, previous cardiac operations, and immunologic disorders.

Four patients had a history of controlled chronic obstructive pulmonary disease (COPD).

Patient demographics, additional comorbidities, cardiovascular disorders, myocardial functions, surgical procedures, and laboratory measurements are summarized in Table 1. (Table 1) Since oxygen index (OI), $\mathrm{FiO} 2, \mathrm{PaO} 2$, and $\mathrm{PaO} 2 /$ FIO2 ratio are the indicators of ARDS, we followed the levels of oxygenation parameters, postoperatively.

Surgical approaches: Patients were pre-medicated using

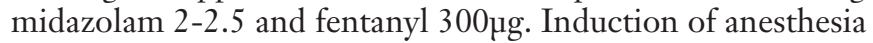
was provided by using midazolam $0.2 \mathrm{mg} / \mathrm{kg}$ and pancuronium $0.1 \mathrm{mg} / \mathrm{kg}$. Anesthesia was maintained with continuous infusion of midazolam, fentanyl, pancuronium, and sevoflurane. We used off-pump ( $N=15 ; 31.5 \%)$ and on-pump cardiac procedures $(N=33)$.

Twenty-seven patients had an isolated CAD $(N=27$; $56.2 \%)$, and seven patients had an isolated valvular disease. CABG accompanied with heart valve surgery was performed in 10 patients. Four CABG patients were accompanied with unilaterally severe carotid artery stenosis. Hemodynamic status including the heart rate, the mean arterial pressure
(MAP), cardiac output $(\mathrm{CO})$, cardiac index $(\mathrm{CI})$, and mean pulmonary artery pressure (PAP) were recorded. Extracorporeal circulation was initiated. We administered antegrade cold blood cardioplegia. We performed CABG and valve surgeries using a traditional method using the left and/or the right internal thoracic artery (ITA) and saphenous vein (SV).

Coronary artery bypass grafting concomitant with valve surgery was performed in 10 patients. An isolated mitral valve replacement (MVR) $(N=4)$ and an isolated aortic valve replacement (AVR) $(N=2)$ were performed in six patients $(14.5 \%)$. We performed a double valve replacement in one patient, who had severe aortic stenosis and mitral valve stenosis $(2 \%)$. In patients, whose age was $\geq 70$ years, and with a small aortic root, we preferred suture-less aortic valve implantation for shortening the duration of the operation and the ECC time.

Off-pump CABG technique was performed in isolated CABG $(N=11 ; 22.9 \%)$ and in patients who required concomitant CEA $(N=4 ; 8.3 \%)$. Minimally invasive direct coronary artery revascularization (MID-CAB) was performed through left anterior thoracotomy incision in four off-pump CABG surgeries $(8.3 \%)$. Mini J-sternotomy or a right anterior thoracotomy incision was performed in patients with an isolated AVR or mitral valve surgery $(N=6 ; 12.5 \%)$.

In our 48 patients confirmed with Covid-19 shortly after surgery, we observed low levels in $\mathrm{PO} 2$ and oxygen saturation $(\mathrm{SaO} 2)$ as well as a serious decrease in $\mathrm{FiO} 2$ and $\mathrm{PaO} 2 / \mathrm{FiO} 2$ ratios. We detected a dramatic decrease in the oxygenation saturation indexes (OSI) and the increase in mean airway pressure in reintubated patients. We recorded arterial blood gas analyses, arterial $\mathrm{PH}$, postoperative BUN, and creatinine levels after surgery. Failure of extubation, re-intubation due to respiratory dysfunction, ICU readmission, postoperative c-Tn-I and CK-MB release, blood lymphocyte levels, $\mathrm{D}$-dimer, hs-CRP, and fibrinogen levels were analyzed.

The primary findings were in-hospital mortality. The secondary findings were readmission to ICU, requirement of intubation, hospitalization time, and end-organ failure. According to previous description, we classified the level of ARDS as mild, moderate, and severe using a thoracic CT image.

Statistical analysis: The statistical analysis was performed using automated SPSS software version 19 (SPSS Inc. Chicago, IL) and SAS. If categorical variables were not normally distributed between patients who were readmitted to the ICU with severe respiratory distress and those who were not, then they were compared using the Chi-Square and Mann-Whitney $\mathrm{U}$ tests, and the results were expressed as percentages. Continuous variables were presented as median with interquartile range (IQR). Variables were compared using the Chi-Square or the Fisher's exact test, as required. Fisher's exact test was used for the comparison of the survived and non-survived patients. All $P$-values were two-tailed, and a $P \leq 0.05$ was accepted as significant.

\section{RESULTS}

Extubation after surgery was performed uneventfully in these 48 patients in the first day after surgery. The median intubation time was 11.6 \pm 3.2 hours (interquartile range: 5-17 
Table 1. Characteristics of patients undergoing on-pump and off-pump patients.

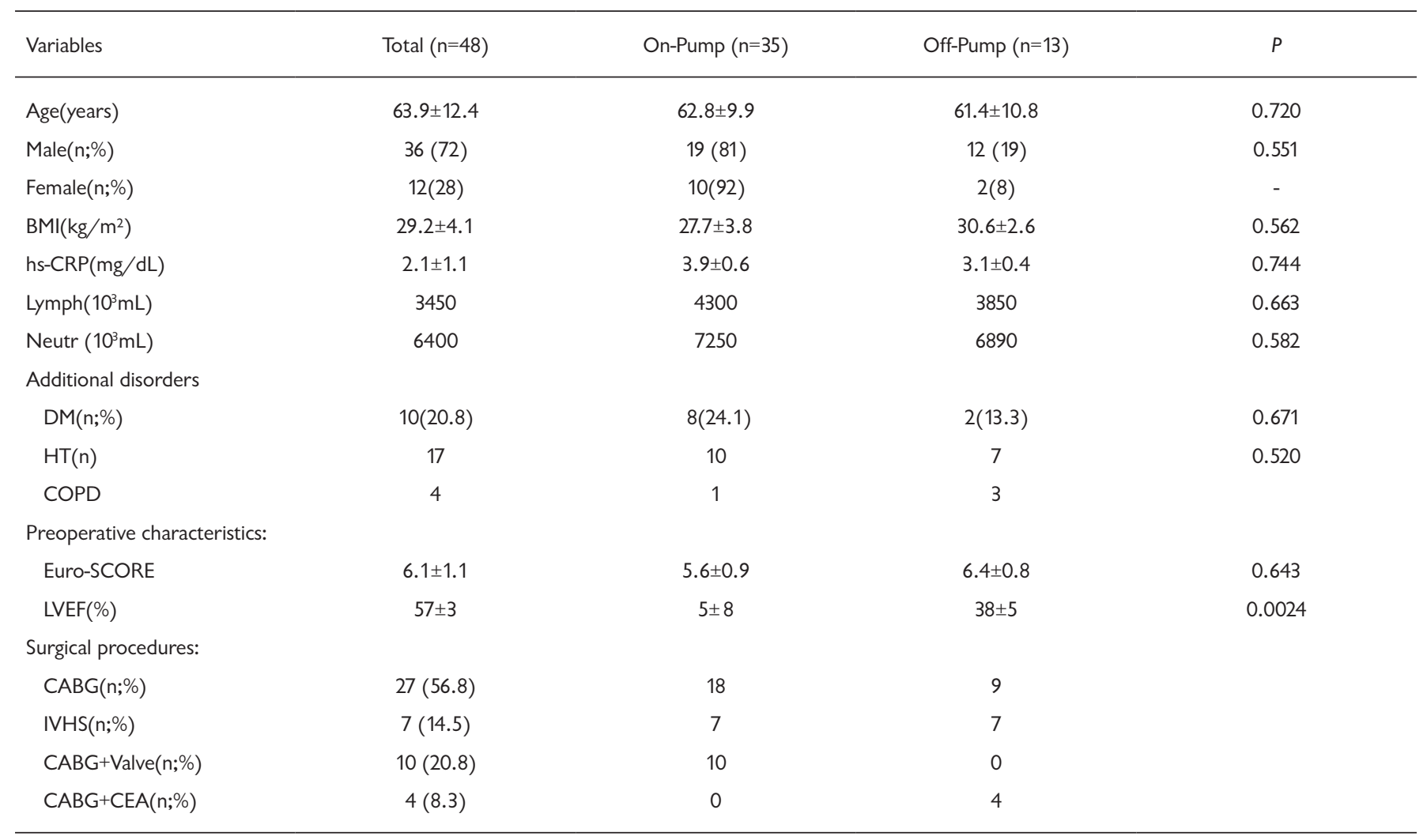

Data are presented as mean \pm SD.

BMI: Body mass index; hs-CRP: high sensitive CRP; Lymph: lymphocyte; Neutr: neutrophil; DM: diabetes mellitus; HT: hypertension; COPD: chronic obstructive pulmonary disease; LVEF: left ventricular ejection fraction; CPB: extracorporeal circulation; ACC: aortic cross-clamp; CABG: coronary artery by-pass grafting; IVHS: isolated valvular heart surgery; CEA: Carotis endarterectomy.

hours). The median time from cardiac surgery to Covid-19 diagnosis was $8.4 \pm 2.7$ days (range: 2-18 days). The major symptoms of patients were dyspnea, fever, and cough. Hypoxia after weaning from the respirator was observed in 18 patients (IQR: $70-93 \%)$. The median time from ICU readmission to death was $23.4 \pm 9.6$ days (IQR: $8-73$ days).

No evidence of pleural effusion or tamponade was detected. No sternal dehiscence or bacterial infection were found. Intraoperative and postoperative data and mediastinal bleeding after surgery are summarized in Table 2. (Table 2)

While the RT-PCR test result was positive in 29 patients, it was negative in the remaining 19 patients, who had a low oxygen saturation [mean: $85 \pm 6 \%$ (range: $74-90$ )]. Four out of 29 Covid-19 confirmed patients were from the off-pump group (13.7\% vs. $86.3 \%$ ). (Table 3 ) Computed tomography showed that there was the presence of mild $(N=9)$, mild to moderate $(N=7)$, and serious ARDS $(N=3)$ with typical lung involvement, due to Covid-19 in 19 patients who had a negative RT-PCR test. (Figure 1) (Figure 2)

Ground-glass opacities with or without pleural thickening, localized or diffuse bilateral pulmonary consolidation (Figure 3 ), and with or without bronchiectasis frequently were observed, according to thoracic CT (Figure 4). (Figure 3) (Figure 4)
We readmitted 11 patients to the ICU because they had severe hypoxia and ARDS (22.6\%). We intubated eight out of 11 patients. The median time from ICU readmission to intubation time was $4.1 \pm 0.9$ days (IQR: $1-7$ days). The mean age of those eight intubated patients was $81 \pm 11$ years. The median $\mathrm{PaO}_{2} / \mathrm{FiO}_{2}$ ratio and OSI in eight patients were $\leq 200$ and $11.3 \pm 4.3$, respectively.

High-flow nasal oxygenation therapy was initiated. We administered remdesivir $(N=48 ; 100 \%)$, corticosteroid (dexamethasone; $N=28 ; 58.3 \%$ ), nonsteroidal anti-inflammatory $\operatorname{drug}(N=41 ; 85.4 \%)$, and bronchodilators $(N=44 ; 91.6 \%)$, as well as intravenous antibiotics (moxifloxacin or azithromycin). To prevent intracoronary or deep venous thrombosis, we prescribed aspirin (daily dose of $100 \mathrm{mg}$ ) and low molecular weight heparin (LMWH).

In the readmitted patients, $\mathrm{SaO}_{2}, \mathrm{FiO}_{2}$, and $\mathrm{PaO}_{2}$ levels were significantly low when compared with non-admitted patients. Neutrophil and lymphocyte count in non-readmitted patients were $9.27 \pm 4.78 \times 10^{3} / \mathrm{ml}$ and $3.73 \pm 0.64 \times 10^{3} /$ $\mathrm{ml}$, respectively. However, blood analyses showed that neutrophil and lymphocyte levels were $14.77 \pm 6.79 \times 10^{3} / \mathrm{ml}$ and $2.87 \pm 0.02 \times 10^{3} / \mathrm{ml}$ in patients, who were readmitted to the ICU. Neutrophil/Lymphocyte ratio in readmitted patients 
Table 2. Intraoperative and early postoperative characteristics of patients confirmed with Covid-19.

\begin{tabular}{|c|c|c|c|c|}
\hline CPB time (min.) & $97 \pm 49$ & $97 \pm 49$ & - & - \\
\hline $\mathrm{PCl}(\mathrm{n}: \%)$ & 6 & 4 & 2 & 0.067 \\
\hline No of grafts in $C A B G$ & $3.1 \pm 1.6$ & $3.5 \pm 1.2$ & $1.9 \pm 0.3$ & 0.022 \\
\hline $\mathrm{NE}(\mu \mathrm{g} / \mathrm{kg} / \mathrm{min})$. & $0.02 \pm 0.03$ & $0.01 \pm 0.02$ & $0.03 \pm 0.03$ & 0.430 \\
\hline $\mathrm{DA}(\mu \mathrm{g} / \mathrm{kg} / \mathrm{min})$. & $0.20 \pm 0.11$ & $0.24 \pm 1.10$ & $0.30 \pm 0.9$ & 0.672 \\
\hline Dobutamine $(\mu \mathrm{g} / \mathrm{kg} / \mathrm{min})$. & $0.03 \pm 2.84$ & $1.19 \pm 2.69$ & $3.01 \pm 3.56$ & 0.252 \\
\hline \multicolumn{5}{|l|}{ Mediastinal bleeding $(\mathrm{mL})$} \\
\hline POD1 & $550(270-1000)$ & $640(490-660)$ & $240(75-220)$ & 0.009 \\
\hline
\end{tabular}

Data are presented as mean SD, (n;\%).

CBP: cardiopulmonary by-pass; ACC: aortic cross-clamp time; PCl; percutaneous coronary intervention; CABG: coronary artery by-pass grafting; NE: Norepinephrine; DA:dopamine; POD: postoperative day; PRBCs: Package red blood cells; ICU: intensive care unit.

Table 3. Clinical data and respiratory functions of 48 patients in the early postoperative period before the diagnosis of Covid-19.

\begin{tabular}{lcccc}
\hline Variables & Total $(\mathrm{n}=48)$ & On-Pump $(\mathrm{n}=33)$ & Off-Pump $(\mathrm{n}=15)$ & $P$ \\
\hline $\mathrm{SpO}_{2}(\%)$ & $97(93-100)$ & $96(93-100)$ & $98(97-100)$ & 0.834 \\
$\mathrm{FiO}_{2}(\%)$ & $0.58 \pm 0.2$ & $0.56 \pm 0.02$ & $0.59 \pm 0.02$ & 0.850 \\
$\mathrm{PaO}_{2}\left(\mathrm{mmHg}^{2}\right)$ & $119 \pm 11$ & $114 \pm 32$ & $125 \pm 13$ & 0.872 \\
$\mathrm{PaO}_{2} / \mathrm{FiO}_{2}(\mathrm{Ratio})$ & $297 \pm 60$ & $286 \pm 86$ & $305 \pm 56$ & 0.567 \\
$\mathrm{PEEP}\left(\mathrm{cmH}_{2} \mathrm{O}\right)$ & $7.09 \pm 2.1$ & $5.9 \pm 0.5$ & $6.2 .1 \pm 2.2$ & 0.723 \\
$\mathrm{AP}\left(\mathrm{cmH}_{2} \mathrm{O}\right)$ & $9.8 \pm 2.6$ & $10.2 \pm 1.2$ & $9.8 \pm 1.4$ & 0.794 \\
\hline
\end{tabular}

$\mathrm{SpO}_{2}$ : Oxygen saturation; $\mathrm{FiO}_{2}$ : Fractionated inspired oxygen; $\mathrm{PaO}_{2}$ : Partial oxygen pressure. PEEP: positive end-expiratory pressure; AP: airway pressure.

was significantly high when compared with non-admitted patients $(2.48 \pm 2.76$ vs. $5.17 \pm 0.07)(P=0.001)$. (Table 5)

The median d-dimer levels in readmitted and non-admitted patients were $480 \pm 210 \mu \mathrm{g} / \mathrm{L}$, and $1756 \pm 522 \mu \mathrm{g} / \mathrm{L}$, respectively $(P=0.0001)$. Hs-CRP levels were significantly high in readmitted patients $(4.08 \pm 1.20 \mathrm{mg} / \mathrm{dL}$ vs. $13.2 \pm 7.30 \mathrm{mg} / \mathrm{dL})$ $(P=0.001)$.

\section{DISCUSSION}

To our knowledge, we presented the first report of postoperative ARDS related to Covid-19 activation after open-heart surgery in a limited number but an important asymptomatic cohort from five tertiary centers. Previous investigations showed ARDS after cardiac and non-cardiac surgeries with
Covid-19 and have been published in the literature [Barkhordari 2020; Lei; Aminian 2020; Niknam 2020]. Barkhordari et al. reported ARDS in 25 asymptomatic Covid-19 cardiac surgery patients who underwent urgent cardiac surgery [Barkhordari 2020]. The rate of mortality in readmitted patients was $16 \%$ in Barkhordari's series. Nicknam et al. proposed prospective research for better understanding of the heterogeneity of Covid-19 positive patients who underwent cardiac surgery for understanding the decision in the pandemic period [Niknam 2020]. Shi et al. showed the relationship between cardiac injury include myocarditis with mortality in patients with Covid-19 [Shi 2020]. Our study demonstrated that urgent PCI and CABG in Covid-19 patients might be performed with acceptable mortality in selected patients [Keskin 2021]. Keskin et al. proposed that elective cardiovascular surgery and PCI might be performed to decrease 


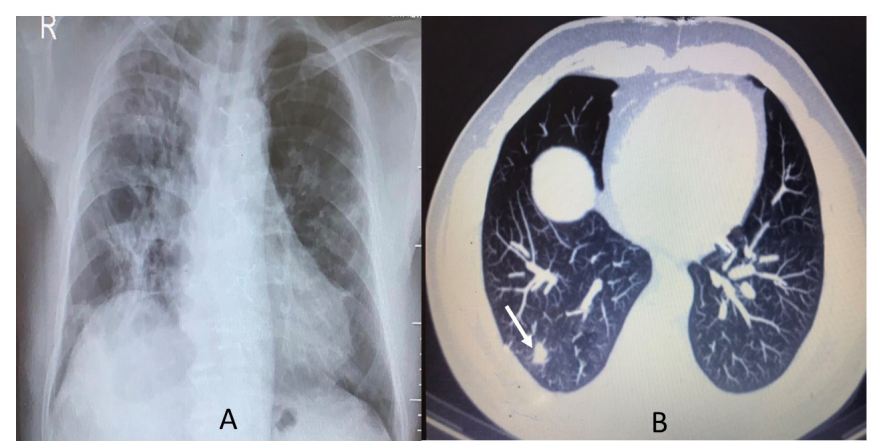

Figure 1. Postoperative chest $\mathrm{x}$-ray after readmission to intensive care unit $(A)$, and thoracic computed tomography $(B)$ shows a pneumonic infiltration after coronary artery bypass grafting surgery in a 75-year-old male patient who had hypertension and diabetes mellitus. The white arrowhead demonstrates a local consolidation in the right lower lobe. Thoracic computed tomography also shows diffuse abnormal vascularization in both lungs.

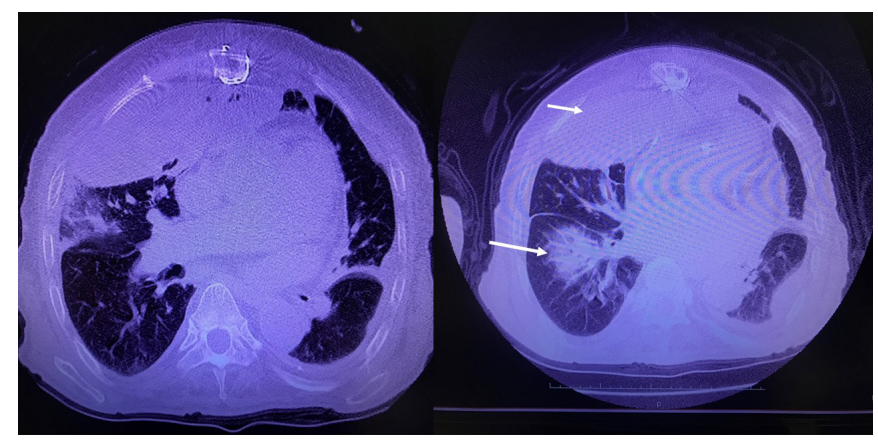

Figure 3. This figure exhibits pneumonic infiltration in the right lower lobe and diffuse ground glass opacities with pleural thickening in a patient, who is undergoing coronary artery bypass grafting surgery in the 8th day after surgery. This patient was readmitted to the intensive care unit because severe hypoxic situation related to acute respiratory distress and hemodynamic instability. This patient died in the 28th day, due to multiorgan failure (white arrowheads demonstrate pleural fluids, and localized, but a large consolidation).

mortality due to cardiovascular events [Keskin 2021]. In addition, they suggested randomized clinical articles about these unique patients [Keskin 2021]. We demonstrated that there were negative effects of ECC on immunologic situations because it decreased the circulating lymphocytes after cardiac surgery [Bayer 2009]. Therefore, many factors are playing the role of clinical outcomes after cardiac surgery. Myocarditis associated with cardiac dysfunction and arrhythmias is a significant factor of mortality in Covid-19 patients by Guo [Guo 2020]. According to previous studies, ARDS accompanied with myocardial inflammation may be the potential mechanism of high mortality.

We suggest thoracic CT in postoperative symptomatic patients after cardiac surgery because it is a more sensitive tool than RT-PCR in the situation of pulmonary involvement [Fang 2020]. We demonstrated diffuse or localized typical Covid-19 findings using thoracic CT in a number of patients.
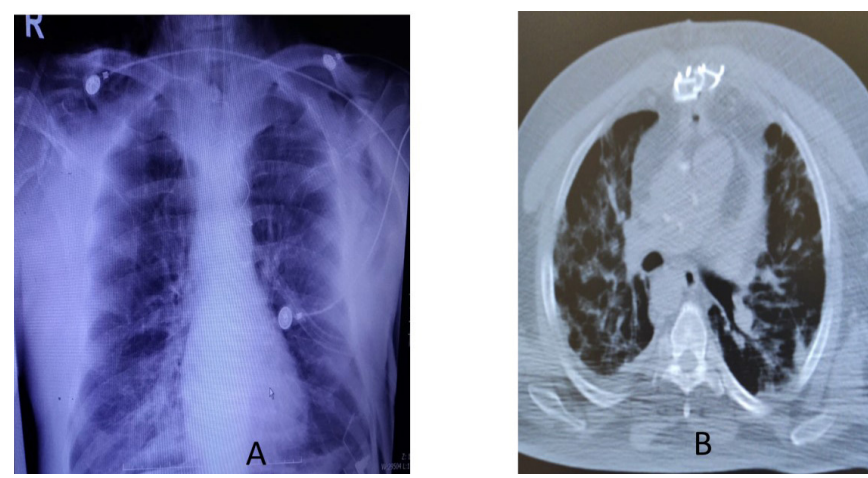

Figure 2. Chest $x$-ray (A) and Thoracic computed tomography (B) in the 5 th day of surgery demonstrates postoperative bilateral pneumonic infiltration in a 47-year-old male patient who underwent double valve replacement. According to thoracic computed tomography, there are bilateral pleural thickening and diffuse consolidation related to Covid-19 infection (B).

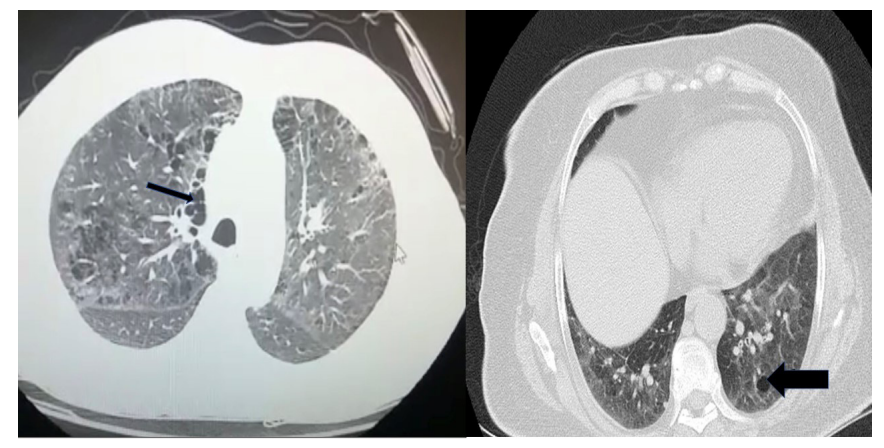

Figure 4. Thoracic computed tomography demonstrates bilateral diffuse pneumonic infiltration accompanied with diffuse bronchiectasis (black arrowheads) in a 63-year-old patient, who had an initial negative reverse transcriptase polymerase chain reaction test. Bilateral pneumonic infiltration is detected in the 7th day after their coronary artery bypass graft operation. The patient died related to multiorgan failure related to acute respiratory distress.

According to our cohort, after readmission to the ICU, the rate of intubation and mortality were high in our patients who had diffuse pneumonic infiltration.

We analyzed the baseline patients' characteristics, blood neutrophil, lymphocyte counts, d-dimer, and fibrinogen; fibrinogen and d-dimer levels are predictors of mortality and morbidity [American College of Surgeons 2020; American College of Surgeons 2020; McAloon 2020; Velly 2020; Driggin 2020]. While blood neutrophil counts increased significantly, the lymphocyte levels decreased in a number of patients. When compared with the ratio of neutrophil/ lymphocyte in readmitted patients, it decreased significantly $(2.48 \pm 1.76$ vs. $5.17 \pm 2.07)$.

Acute lung injury related to post-cardiotomy Covid-19 activation in our series was almost three to four times more, when compared with the pre-pandemic period [American College of Surgeons 2020; McAloon 2020; Velly 2020]. In urgent or emergency situations, hybrid approaches (CABG 
Table 4. Clinical data of non-admitted and readmitted patients confirmed with Covid-19 who had respiratory symptoms.

\begin{tabular}{|c|c|c|c|}
\hline $\mathrm{n} ; \%$ & $37(77.1)$ & $11(22.9)$ & 0.0000 \\
\hline Mortality(n;\%) & $1(2.7)$ & $6(54.5)$ & 0.0001 \\
\hline $\operatorname{ARF}(n ; \%)$ & $1(2.7)$ & $2(18.1)$ & 0.0001 \\
\hline Arrhythmia & $3(8.1)$ & $4(36.3)$ & 0.0022 \\
\hline $\mathrm{SpO}_{2}(\%)$ & $90(82-93)$ & $77(69-88)$ & 0.022 \\
\hline $\mathrm{FiO}_{2}(\%)$ & $0.77 \pm 0.14$ & $0.99 \pm 2.44$ & 0.002 \\
\hline $\mathrm{PaO}_{2}(\mathrm{mmHg})$ & $121 \pm 33$ & $67 \pm 11$ & 0.030 \\
\hline $\mathrm{PaO}_{2} / \mathrm{FiO}_{2}$ & $211 \pm 36$ & $99 \pm 90$ & 0.001 \\
\hline ICU time (hour) & - & $169 \pm 22$ & - \\
\hline
\end{tabular}

Table 5. Biochemical analyses of non-readmitted and readmitted patients.

\begin{tabular}{|c|c|c|c|}
\hline $\operatorname{Neu}\left(10^{3} / \mathrm{ml}\right)^{*}$ & $9.27 \pm 4.78$ & $4.77 \pm 6.79$ & 0.002 \\
\hline Neu/Lymph* & $2.48 \pm 1.76$ & $5.17 \pm 2.07$ & 0.001 \\
\hline Fibrinogen $(\mathrm{mg} / \mathrm{dL})^{*}$ & $550 \pm 245$ & $850 \pm 580$ & 0.000 \\
\hline $\mathrm{hs}-\mathrm{CRP}(\mathrm{mg} / \mathrm{dL})^{*}$ & $4.08 \pm 1.20$ & $13.2 \pm 7.30$ & 0.001 \\
\hline
\end{tabular}

Data are presented as mean SD.

ARF: acute renal failure; LOS: low out-put syndrome; hs-CRP: highly sensitive C-reactive protein; PEEP: positive end-expiratory pressure; AP: airway pressure; $\mathrm{PaO}_{2}$ : pressure of inspiratory oxygen; $\mathrm{FiO}_{2}$ : fractionated inspiratory oxygen; OSI: oxygenation saturation index; ICU: Intensive care unit. Neu: neutrophil; Lymph: lymphocyte.

following PCI) using a beating heart to prevent ECC in select patients or MID-CAB procedures may be decided during the pandemic period. Because there are no scientific investigations about these technical considerations, we need randomized studies to compare traditional methods.

The incubation period of Covid-19 has been reported as two to 10 days [American College of Surgeons 2020; American College of Surgeons 2020]. In another study, the authors showed that pooled parameters estimated the median incubation period was 11.7 days (95\% CI) [McAloon 2020; Velly 2020]. An infected, asymptomatic person may transmit Covid-19 to our cohort just before the operation. The median $\mathrm{PaO}_{2} / \mathrm{FiO}_{2}$ in non-admitted patients who had unilateral or with bilateral local opacities was $265 \pm 44$. Of 37 patients, acute lung injuries were classified as mild, mild to moderate, and severe. Periodic radiologic examinations showed normal chest $\mathrm{x}$-rays 10 days later.
According to Delbove et al. [Delbove 2021], we administered high flow nasal cannula to increase saturation of $\mathrm{O}_{2}$.

Our experiences demonstrated that oxygen saturation index, $\mathrm{FiO} 2$, and $\mathrm{PaO} 2$ may be used as the first signs of Covid19 activation after cardiac surgery. Thoracic CT may support confirmation of Covid-19 and showed the degree of ARDS. The incubation period of Covid-19 can extend to critical levels of virus replication within hours or days, depending on the patient's status (older age, comorbidity, longer ECC, etc.). Negative RT-PCR test may be observed since the viral RNA does not reach detectable thresholds at the onset of the disease. We hypothesized that the physiologic stressors, atelectasis, and proinflammatory may trigger Covid-19 replication.

The study of the Extracorporeal Life Support Organization Registry included 1035 Covid-19 patients, who received extracorporeal membrane oxygenation (ECMO) support. 
The authors showed that overall incidence of in-hospital mortality was $37 \%$ in patients who received ECMO. Tidal volumes $(6-8 \mathrm{~mL} / \mathrm{kg})$, PEEP, and appropriate ventilatory parameters with hemodynamic stability should be adjusted on the basis of tissue oxygenation. We suggest that perioperative acid-base management and protection from hypercapnia may warrant good respiratory outcomes after surgery in mild-tomoderate Covid-19 patients [Barbaro 2020].

\section{CONCLUSION}

According to our clinical experiences, there can be varying degrees of ARDS development, due to Covid-19 activation after heart surgery if the virus is in the latent period. Similar scientific reports will create an important awareness for surgeons and attract the attention of surgeons. Postoperative course of cardiac surgery may be complicated, due to Covid-19 activation with a high readmission to ICU and mortality rate. Negative RT-PCR test before cardiac surgery and absence of pulmonary dysfunction do not guarantee postoperative Covid-19 infection activation. In this rare situation, we may encounter negative consequences, and high mortality rates related to serious pulmonary complications and ARDS.

\section{REFERENCES}

American College of Surgeons. 2020. COVID-19: guidance for triage of non-emergent surgical procedures. https:// www.facs.org/covid-19/ clinical-guidance/triage

American College of Surgeons. 2020. COVID-19: guidelines for triage of cardiac surgery patients. https://www.facs.org/ covid-19/ clinical-guidance/elective-case/cardiac-surgery

Aminian A, Safari S, Razeghian-Jahromi A, Ghorbani M, Delaney CP. 2020. COVID-19 outbreak and surgical practice. Ann Surg. 272(1):27-29.

Bangalore S, Sharma A, Slotwiner A, Yatskar L, Harari R, Shah B, et al. 2020. ST-Segment Elevation in Patients with Covid-19-A. Case Series. N Engl J Med. 382:2478-80.

Barbaro RP, MacLaren G, Boonstra PS, Iwashyna TJ, Slutsky AS, Fan E, et al. 2020. Extracorporeal membrane oxygenation support in COVID19: an international cohort study of the Extracorporeal Life Support Organization registry. Lancet. (10):396:1071-1078.

Barkhordari K, Khajavi MR, Bagheri J, Nikkhah S, Shirzad M, Barkhordari S, Kharazmian K, Nosrati M. 2020. Early respiratory outcomes following cardiac surgery in patients with COVID-19. J Card Surg. 35(10):2479-2485.

Bayer A, Dogan OF, Ersoy F, Ersoy U. 2009. The effect of open heart surgery on circulating lymphocytes and lymphocyte subsets in pediatric patients. Turk J Thorac cardiovasc Surg. 17(1):13-17.

Chen N, Zhou M, Dong X, et al. 2020. Epidemiological and clinical characteristics of 99 cases of 2019 novel coronavirus pneumonia in Wuhan, China: a descriptive study. Lancet. 395:507-513.

Chieffo A, Stefanini GG, Price S, et al. 2020. EAPCI position statement on invasive management of acute coronary syndromes during the
COVID-19 pandemic. Eur Heart J. 41:1839-851.

Curzen N. 2020. An extended statement by the British Cardiovascular Intervention Society president regarding the COVID-19 pandemic. Interv Cardiol Rev. 15:01.

Delbove A, Foubert A, Mateos F, Guy T, Gousseff M. 2021. High flow nasal cannula oxygenation in COVID-19 related acute respiratory distress syndrome: a safe way to avoid endotracheal intubation? Ther Adv Respir Dis. 15: 1-10.

Driggin E, Madhavan MV, Bikdeli B, Chuich T, Laracy J, Biondi-Zoccai G, et al. 2020. Cardiovascular Considerations for Patients, Health Care Workers, and Health Systems During the COVID-19 Pandemic. J Am Coll Cardiol. 12;75(18):2352-2371.

Fang Y, Zhang H, Xie J, et al. 2020. Sensitivity of chest CT for COVID19: comparison to RT-PCR. Radiol. 19:432.

Giulio G. Montorfano SM, Trabattoni D, et al. 2020. ST-Elevation Myocardial Infarction in Patients with COVID-19: Clinical and Angiographic Outcomes. Circulation. 141:2113-16.

Guo T, Fan Y, Chen M, Wu X, Zhang L, He T, et al. 2020. Cardiovascular Implications of Fatal Outcomes of Patients With Coronavirus Disease 2019 (COVID-19). JAMA Cardiol. 5(7):811-18.

Ing ZC, Zhu HD, Yan XW, Chai WZ, Zhang S. 2020. Recommendations from the Peking Union Medical College Hospital for the management of acute myocardial infarction during the COVID-19 outbreak. Eur Heart J. 41:1791-94.

Keskin G, Khalil, Uysal A. 2021. Should We Postpone Elective Cardiovascular Procedures and Percutaneous Coronary Interventions During the COVID-19 Pandemic? Heart Surg Forum. 24(1): 22-030.

Keskin G, Uysal A, Erturk E, Hafiz E, Dogan OF. 2021. Urgent Percutaneous Coronary Artery Intervention and Coronary Artery Bypass Grafting in STEMI Patients with Confirmed COVID-19. Heart Surg Forum. 23;24(3):564-574.

Lei S, Jiang F, Su W, et al. Clinical characteristics and outcomes of patients undergoing surgeries during the incubation period of COVID-19 infection. E Clinical Medicine.

McAloon C, Collins A, Hunt K, Barber A, Byrne AW, Butler F, et al. 2020. Incubation period of COVID-19: a rapid systematic review and meta-analysis of observational research. BMJ Open. 16;10(8):e039652.

Niknam J, Rong LQ. 2020. Asymptomatic patients with coronavirus disease and cardiac surgery: When should you operate? J Card Surg. 1-3.

Shi S, Kin B, Bo S. 2020. Association of cardiac injury with mortality in hospitalized patients with COVID-19 in Wuhan, China. JAMA Cardiol. JAMA Cardiol. 5(7):802-810.

Velly L, Gayat E, Quintard H, Weiss E, Jong AD, Cuvillon P, Audibert G. 2020. Guidelines: Anaesthesia in the context of COVID-19 pandemic. Anaesth Crit Care Pain Med. 39(3):395-415.

Welt FGP, Shah PB, Aronow HD, Bortnick AE, Henry TD, Sherwood MW, Young MN, Davidson LJ, Kadavath S, Mahmud E, et al. 2020. American College of Cardiology's Interventional Council and the Society for Cardiovascular Angiography and Interventions. Catheterization laboratory considerations during the coronavirus (COVID-19) pandemic: from the ACC's Interventional Council and SCAI. J Am Coll Cardiol. 75:2372-2375.

World Health Organization. 2019. Coronavirus disease (COVID-19) Pandemic. https://www.who.int/emergencies/diseases/novel-coronavirus-2019. 